\title{
Cooperative Control of Linear Multi-Agent Systems via Distributed Output Regulation and Transient Synchronization
}

\author{
Georg S. Seyboth ${ }^{\mathrm{a}}$, Wei Ren ${ }^{\mathrm{b}}$, Frank Allgöwer ${ }^{\mathrm{a}}$ \\ anstitute for Systems Theory and Automatic Control, University of Stuttgart, Pfaffenwaldring 9, 70550 Stuttgart, Germany. \\ ${ }^{\mathrm{b}}$ Department of Electrical and Computer Engineering, University of California, Riverside, CA 92521, USA.
}

\begin{abstract}
A wide range of multi-agent coordination problems including reference tracking and disturbance rejection requirements can be formulated as distributed output regulation problem. The general framework captures typical tasks such as output synchronization, leader-following, formation keeping, and many more. We present a distributed regulator for groups of identical and non-identical linear agents subject to global external signals affecting all agents as well as local external signals affecting individual agents. Both signal types comprise references and disturbances. The main contribution is a novel coupling among the agents based on their transient state components, or estimates thereof in the output feedback case. The transient synchronization improves the cooperative behavior in transient phases and guarantees a desired decay rate of the synchronization error, which leads to a cooperative reaction on local disturbances acting on individual agents.
\end{abstract}

Key words: Cooperative Control; Distributed control; Multi-agent systems; Regulator theory; Linear output feedback; Synchronization.

\section{Introduction}

In a variety of modern man-made systems, it is desirable to synthesize a cooperative behavior among individual dynamical agents, similar to bird flocks and fish schools observed in nature. Examples include multi-vehicle coordination and formation flight problems, robot cooperation in production lines, power balancing in micro-grids, and many more. Of particular interest are distributed control laws which require only local information exchange between neighboring subsystems and no centralized data collection or processing entity, which provides scalable, flexible, and robust algorithms. A fundamental cooperative control problem is the consensus or synchronization problem for groups of linear dynamical agents. It has been studied extensively over the past decade, starting from single-integrator agents $[19,21]$, to double-integrator agents [20], identical linear agents $[5,15,22,30,31]$, and non-identical linear agents $[6,12,17,32]$.

\footnotetext{
* This research was supported in part by the German Research Foundation (DFG) within the Cluster of Excellence in Simulation Technology (EXC 310/1) at the University of Stuttgart and by the National Science Foundation (NSF) USA under the Grant ECCS1307678.

Email addresses: georg. seyboth@ist.uni-stuttgart.de (Georg S. Seyboth), ren@ee.ucr.edu (Wei Ren), allgower@ist.uni-stuttgart.de (Frank Allgöwer).
}

From a practical point of view, it is desirable to influence the behavior of the group via external reference signals. A solution to this problem is the leader-follower setup $[8,15,18,35]$. The idea is to select a particular agent as leader for the group or introduce a virtual leader and design the distributed control law such that all agents synchronize to this leader. Moreover, it is important to consider external disturbances acting on the multi-agent system, to analyze the performance of the closed-loop system, and to incorporate disturbance rejection or attenuation requirements in the design procedure. Rejection of constant disturbances is addressed in [1,23,34]. In order to tackle both reference and disturbance signals simultaneously, it was proposed in $[11,33]$ to formulate multi-agent coordination problems with external reference and disturbance signals as a synchronized output regulation problem and utilize the classical output regulation theory, cf., $[10,13,28]$. The problem setup in $[11,33]$ consists of an autonomous exosystem and a group of identical linear agents, which are affected by the generalized disturbance signal generated by the exosystem, and a regulation error for each agent which shall converge to zero. The group objective for the multi-agent system is formulated in terms of a common reference signal for all agents and local regulation errors for each agent with respect to the common reference. Cooperation is necessary since not all agents have access to the external signal. By assumption, the group is divided into a group of informed agents which are able to reconstruct the external signal, and uninformed agents which are depen- 
dent on information exchange with informed agents in order to solve their regulation task. The cooperative output regulation problem generalizes existing solutions of the leaderfollower problem [9]. [25, 26] extend the results of [11,33] and present a solution to the cooperative output regulation problem with non-identical agents based on state feedback and output feedback, respectively. Each agent is described by a generalized plant in which all matrices may be different for different agents. The solution proposed in [26] consists of three components: local feedback laws which are constructed based on the classical output regulation theory for each agent; local observers for the state of each agent; and a distributed observer for the generalized disturbance. Besides the distributed observer, this control strategy is decentralized. There are no couplings based on the agent states or outputs and it is inherent in the controller structure that local disturbances are rejected by the affected agent only and no other agent recognizes such a disturbance. This causes the limitation that the group is not able to react in a cooperative manner on local disturbances. In applications such as formation flight and vehicle platooning, keeping a desired formation typically has a higher priority than precisely following a given path with the formation center. A vehicle in a platoon is expected to slow down or accelerate in order to avoid collisions with its follower or predecessor when any of those experiences a disturbance. This fact is also highlighted in [2] and motivates the work in the present paper.

In the present paper, we distinguish between global and local generalized disturbances affecting all or only individual agents in the group, respectively, and present a distributed regulator taking this structure into account. The main contribution is an extension of the distributed regulator with suitable couplings among the agents in order to enable a cooperative reaction of the group on local external disturbances. These couplings allow to impose a desired decay rate on the synchronization error. It is shown that the transient state components of the agents are well suited for this purpose. A recent design method for the coupling gain allows to impose performance specifications such as a minimum decay rate of the synchronization error of the group. Moreover, in a preliminary step, we formulate the cooperative control problem as a single centralized output regulation problem. The solvability conditions for the centralized problem and its particular structure yield necessary and sufficient solvability conditions for the distributed regulation problem.

Outline: Section 2 presents some background and the distributed and centralized output regulation problems. The distributed regulator for general non-identical linear agents is presented in Section 3. In Section 4, the extension of the distributed regulator is derived which guarantees exponential stability of the synchronization error with a desired decay rate. The derivation is based on the assumption that the agents have identical dynamics. A vehicle platooning example illustrates the results. In Section 5, the assumption of identical agent dynamics is relaxed and it is shown how the coupling can be designed for non-identical agents with similar dynamics. Section 6 concludes the paper.

\section{Problem Setup}

Notation: The open left half plane, imaginary axis, and open right half plane of $\mathbb{C}$ are denoted by $\mathbb{C}^{-}, \mathbb{C}^{0}$, and $\mathbb{C}^{+}$, respectively. For $z \in \mathbb{C}, \bar{z}$ is the complex conjugate, $\operatorname{Re}(z)$ is the real part and $\operatorname{Im}(z)$ is the imaginary part. The spectrum of $A \in$ $\mathbb{C}^{n \times n}$ is denoted by $\sigma(A) \subset \mathbb{C} \cdot \operatorname{diag}\left(M_{k}\right)=\operatorname{diag}\left(M_{1}, \ldots, M_{N}\right)$ and $\operatorname{stack}\left(M_{k}\right)$ denote a block diagonal matrix and a vertical stack of matrices with blocks $M_{k}, k=1, \ldots, N$, respectively. For a set of vectors $v_{k} \in \mathbb{R}^{n}, k=1, \ldots, N, v \in \mathbb{R}^{N n}$ denotes the stack vector $v=\left[v_{1}^{\top} \cdots v_{N}^{\top}\right]^{\top}$. For $v \in \mathbb{R}^{n}, \operatorname{diag}(v)$ is a diagonal matrix with the entries of $v$ on the diagonal. The identity matrix of dimension $N$ is $I_{N}$ and the vector of ones is 1. For a transfer function matrix $G,\|G\|_{\infty}$ denotes its $\mathcal{H}_{\infty}$ norm. The symbol $\otimes$ denotes the Kronecker product. The pairs $(A, B),(A, C)$ are called stabilizable, detectable with decay rate $\gamma>0$, if the decay rate of the uncontrollable, unobservable modes is at least $\gamma$, i.e., $\left(A+\gamma I_{n}, B\right),\left(A+\gamma I_{n}, C\right)$ are stabilizable, detectable, respectively. Any matrix $L$ such that $A-L C$ is Hurwitz is called an observer gain matrix.

Agent models: The dynamics of the non-identical agents are described by linear state-space models. The agent index set is defined as $\mathcal{N}=\{1, \ldots, N\}$, where $N$ is the number of agents in the group. The dynamics of the undisturbed agents are described by $\dot{x}_{k}=A_{k} x_{k}+B_{k} u_{k}$, where $x_{k}(t) \in \mathbb{R}^{n_{k}^{x}}$ is the state and $u_{k}(t) \in \mathbb{R}^{n_{k}^{u}}$ is the control input of agent $k \in \mathcal{N}$. The cooperative control problem is formulated in terms of the generalized plant

$$
\begin{aligned}
& \dot{x}_{k}=A_{k} x_{k}+B_{k} u_{k}+B_{k}^{d^{g}} d^{g}+B_{k}^{d^{\ell}} d_{k}^{\ell} \\
& y_{k}=C_{k} x_{k}+D_{k} u_{k}+D_{k}^{d^{g}} d^{g}+D_{k}^{d^{\ell}} d_{k}^{\ell} \\
& e_{k}=C_{k}^{e} x_{k}+D_{k}^{e} u_{k}+D_{k}^{e d^{g}} d^{g}+D_{k}^{e d^{\ell}} d_{k}^{\ell}
\end{aligned}
$$

where $y_{k}(t) \in \mathbb{R}^{n_{k}^{y}}$ is the measurement output of agent $k$ and $d^{g}(t) \in \mathbb{R}^{n^{d^{g}}}, d_{k}^{\ell}(t) \in \mathbb{R}^{n_{k}^{d^{\ell}}}$ are external signals specified next. The regulation error $e_{k}(t) \in \mathbb{R}^{n_{k}^{e}}$ is defined such that asymptotic tracking and disturbance rejection is equivalent to $e_{k}(t) \rightarrow 0$ as $t \rightarrow \infty$ for all initial conditions.

External Signals: Two types of external input signals affecting the group are considered: a global signal that affects all agents and local signals that affect individual agents in the group. Each of these signals represents a generalized disturbance which may consist of reference signals and disturbances. The global signal $d^{g}(t), t \geq 0$, is a solution of

$$
\dot{d}^{g}=S^{g} d^{g},
$$

called global exosystem, where $\sigma\left(S^{g}\right) \subset \mathbb{C}^{0} \cup \mathbb{C}^{+}$. The local signal $d_{k}^{\ell}(t), t \geq 0$, is a solution of the local exosystem system

$$
\dot{d}_{k}^{\ell}=S_{k}^{\ell} d_{k}^{\ell}
$$

where $\sigma\left(S_{k}^{\ell}\right) \subset \mathbb{C}^{0} \cup \mathbb{C}^{+}$, for all $k \in \mathcal{N}$.

Remark 1 The exosystems (2), (3) could be combined into a single large exosystem generating all disturbances acting 
on the group. In that sense, the present formulation does not enlarge the problem class compared to [26,27]. The main benefit of taking the structure of the exosystem explicitly into account is the decomposition of the regulator equations and the reduction of the controller dimension, as we will see later in Lemma 1 and Theorem 3, respectively.

Information Structure: All agents have communication capabilities. The communication topology is described by a directed graph $\mathcal{G}=(\mathcal{N}, \mathcal{E})$, with node set $\mathcal{N}=\{1, \ldots, N\}$ corresponding to the agent index set and edge set $\mathcal{E} \subset \mathcal{N} \times \mathcal{N}$. A directed edge $(j, k) \in \mathcal{E}$ corresponds to possible information flow from agent $j \in \mathcal{N}$ to agent $k \in \mathcal{N}$. We think of these edges as communication channels. Hence, each agent can transmit local measurements or controller states. The neighbor set of agent $k$ is the subset $\mathcal{N}_{k} \subset \mathcal{N}$ from which $k$ can receive information, i.e., $\mathcal{N}_{k}=\{j \in \mathcal{N}:(j, k) \in \mathcal{E}\}$. We call a directed graph $\mathcal{G}$ connected if it contains a directed spanning tree (which is sometimes called quasi strongly connected in the literature), and strongly connected if there is a directed path from every node to every other node, i.e., every node is the root of a spanning tree. The adjacency matrix $A_{\mathcal{G}}$ of $\mathcal{G}$ is defined element-wise by $a_{k j}=1 \Leftrightarrow(j, k) \in \mathcal{E}$ and $a_{k j}=0$ otherwise. In case $\mathcal{G}$ is connected, its Laplacian matrix $L_{\mathcal{G}}=\operatorname{diag}\left(A_{\mathcal{G}} \mathbf{1}\right)-A_{\mathcal{G}}$ has exactly one zero eigenvalue $\lambda_{1}\left(L_{\mathcal{G}}\right)=0$ and all other eigenvalues $\lambda_{k}\left(L_{\mathcal{G}}\right), k \in \mathcal{N} \backslash\{1\}$, have positive real parts $\operatorname{Re}\left(\lambda_{k}\left(L_{\mathcal{G}}\right)\right)>0$, cf., [21]. In the following, the eigenvalues of $L_{\mathcal{G}}$ are shortly denoted by $\lambda_{k}$, $k \in \mathcal{N}$, and the numbering is always chosen such that $\lambda_{1}=0$. For further details, see $[21,31,32]$ and references therein.

Auxiliary Result: The following result on state synchronization in groups of identical linear systems will be useful.

Theorem 1 ([24]) Consider group of identical linear agents $\dot{x}_{k}=A x_{k}+B u_{k}$ with $x_{k} \in \mathbb{R}^{n_{x}}, k \in \mathcal{N}$, and $(A, B)$ stabilizable. Suppose that $\mathcal{G}$ is connected and consider the static diffusive couplings $u_{k}=K \sum_{j \in \mathcal{N}_{k}}\left(x_{j}-x_{k}\right)$. Let $\mathcal{R} \subset \mathbb{C}^{-}$be an LMI region defined by $\mathcal{R}=\left\{z \in \mathbb{C}: L+z M+\bar{z} M^{\top}<0\right\}$ with some fixed real matrices $L=L^{\top}$ and $M$. If there exist real matrices $Y>0$ and $Z$ such that for all $k \in \mathcal{N} \backslash\{1\}$, the LMIs

$$
L \otimes Y+M \otimes\left(A Y-\lambda_{k} B Z\right)+M^{\top} \otimes\left(A Y-\overline{\lambda_{k}} B Z\right)^{\top}<0
$$

are satisfied, then the coupling gain $K=Z Y^{-1}$ ensures state synchronization: $x_{k}(t)-x_{j}(t) \rightarrow 0$ as $t \rightarrow \infty$ for all $j, k \in \mathcal{N}$ and all initial conditions. Moreover, the poles of all modes corresponding to the synchronization error lie in $\mathcal{R}$.

Theorem 1 is a design method for the coupling gain $K$ which takes performance specifications in terms of pole placement constraints into account. It utilizes the LMI regions introduced in [4]. Design methods without pole placement constraints are provided in $[15,30,31]$.

The Distributed Output Regulation Problem: The group objective under consideration consists of two parts: asymptotic tracking of reference signals and asymptotic disturbance rejection. Typically, we are interested in synchronization prob- lems which can be formulated as tracking problem of a common, global reference signal. Moreover, our focus will be on the cooperative behavior of the group in transient phases. The distributed output regulation problem is formally stated as follows.

Problem 1 For each $k \in \mathcal{N}$, find a distributed regulator

$$
\begin{aligned}
\dot{z}_{k} & =A_{k k}^{c} z_{k}+B_{k k}^{c} y_{k}+\sum_{j \in \mathcal{N}_{k}}\left(A_{k j}^{c} z_{j}+B_{k j}^{c} y_{j}\right) \\
u_{k} & =C_{k k}^{c} z_{k}+\sum_{j \in \mathcal{N}_{k}} C_{k j}^{c} z_{j}
\end{aligned}
$$

such that the following two conditions are satisfied:

P1) If $d^{g}(0)=0, d_{k}^{\ell}(0)=0, k \in \mathcal{N}$, then, for all initial conditions $x_{k}(0), z_{k}(0)$, it holds that $x_{k}(t) \rightarrow 0$ and $z_{k}(t) \rightarrow 0$ as $t \rightarrow \infty$.

P2) For all initial conditions $d^{g}(0), d_{k}^{\ell}(0), x_{k}(0), z_{k}(0), k \in$ $\mathcal{N}$, it holds that $e_{k}(t) \rightarrow 0$ as $t \rightarrow \infty$.

The Centralized Output Regulation Problem: In case of allto-all communication, (5) becomes a centralized dynamic output feedback controller of the form

$$
\begin{aligned}
\dot{z} & =A^{c} z+B^{c} y \\
u & =C^{c} z .
\end{aligned}
$$

Let $d=\left[\begin{array}{llll}d^{g \top} & d_{1}^{\ell^{\top}} \cdots & d_{N}^{\ell \top}\end{array}\right]^{\top}$. Then, the overall cooperative control problem can be formulated as a single classical output regulation problem by combining all agents (1) into one large generalized plant and one large exosystem as follows:

$$
\begin{aligned}
& \dot{x}=\mathcal{A} x+\mathcal{B} u+\mathcal{B}_{d} d \\
& y=\mathcal{C} x+\mathcal{D} u+\mathcal{D}_{d} d \\
& e=\mathcal{C}_{e} x+\mathcal{D}_{e} u+\mathcal{D}_{e d} d
\end{aligned}
$$

and

$$
\dot{d}=\mathcal{S} d
$$

with the structured matrices $\mathcal{A}=\operatorname{diag}\left(A_{k}\right), \mathcal{B}=\operatorname{diag}\left(B_{k}\right)$, $\mathcal{B}_{d}=\left[\operatorname{stack}\left(B_{k}^{d^{g}}\right) \operatorname{diag}\left(B_{k}^{d^{\ell}}\right)\right], \mathcal{C}=\operatorname{diag}\left(C_{k}\right), \mathcal{D}=\operatorname{diag}\left(D_{k}\right)$, $\mathcal{D}_{d}=\left[\operatorname{stack}\left(D_{k}^{d^{g}}\right) \operatorname{diag}\left(D_{k}^{d^{\ell}}\right)\right], \mathcal{C}_{e}=\operatorname{diag}\left(C_{k}^{e}\right), \mathcal{D}_{e}=\operatorname{diag}\left(D_{k}^{e}\right)$, $\mathcal{D}_{e d}=\left[\operatorname{stack}\left(D_{k}^{e d^{g}}\right) \operatorname{diag}\left(D_{k}^{e d^{\ell}}\right)\right]$ and $\mathcal{S}=\operatorname{diag}\left(S^{g}, \operatorname{diag}\left(S_{k}^{\ell}\right)\right)$. The distributed output regulation problem can only be solved if the centralized output regulation problem has a solution of the form (6). Hence, we study the necessary conditions for the solvability of the overall output regulation problem and exploit the structure in order to derive necessary conditions for the local output regulation problems in the sequel.

Theorem 2 ([10, Thm. 1.14]) Let $(\mathcal{A}, \mathcal{B})$ be stabilizable,

$$
\left(\left[\begin{array}{cc}
\mathcal{A} & \mathcal{B}_{d} \\
0 & \mathcal{S}
\end{array}\right],\left[\begin{array}{ll}
\mathcal{C} & \mathcal{D}_{d}
\end{array}\right]\right)
$$

be detectable, and suppose that $\sigma(\mathcal{S}) \subset \mathbb{C}^{0} \cup \mathbb{C}^{+}$. Then, Problem 1 has a centralized solution (6), if and only if there exists a solution $\Pi, \Gamma$ to the regulator equation

$$
\left[\begin{array}{cc}
\mathcal{A} & \mathcal{B} \\
\mathcal{C}_{e} & \mathcal{D}_{e}
\end{array}\right]\left[\begin{array}{c}
\Pi \\
\Gamma
\end{array}\right]-\left[\begin{array}{c}
\Pi \\
0
\end{array}\right] \mathcal{S}+\left[\begin{array}{c}
\mathcal{B}_{d} \\
\mathcal{D}_{e d}
\end{array}\right]=0
$$


In case all conditions in Theorem 2 are fulfilled, a centralized regulator can be constructed as follows. Choose $\mathbf{F}$ such that $\mathcal{A}-\mathcal{B} \mathbf{F}$ is Hurwitz and $\mathbf{L}$ as an observer gain matrix for (9). Define $\mathbf{G}=\Gamma+\mathbf{F} \Pi$ where $\Pi$, $\Gamma$ solve (10). Then, the controller is given by $u=-\mathbf{F} \hat{x}+\mathbf{G} \hat{d}$ with the observer

$$
\left[\begin{array}{c}
\dot{\hat{x}} \\
\dot{\hat{d}}
\end{array}\right]=\left[\begin{array}{cc}
\mathcal{A} & \mathcal{B}_{d} \\
0 & \mathcal{S}
\end{array}\right]\left[\begin{array}{l}
\hat{x} \\
\hat{d}
\end{array}\right]+\left[\begin{array}{c}
\mathcal{B} \\
0
\end{array}\right] u+\mathbf{L}(y-\hat{y}),
$$

where $\hat{y}=\mathcal{C} \hat{x}+\mathcal{D} u+\mathcal{D}_{d} \hat{d}$. An observer is used since $x$ and $d$ are not directly accessible. This controller is of the form (6) with $z=\left[\begin{array}{ll}\hat{x}^{\top} & \hat{d}^{\top}\end{array}\right]^{\top}$. The control law $u=-\mathbf{F} x+\mathbf{G} d$ is referred to as the full information control law.

It turns out that the solvability condition of (10) decomposes into a set of local regulator equations for the individual agents, as stated next.

Lemma 1 The regulator equation (10) for the overall output regulation problem is solvable, if and only if the local regulator equations

$$
\begin{aligned}
& A_{k} \Pi_{k}^{g}+B_{k} \Gamma_{k}^{g}-\Pi_{k}^{g} S^{g}+B_{k}^{d^{g}}=0 \\
& C_{k}^{e} \Pi_{k}^{g}+D_{k}^{e} \Gamma_{k}^{g}+D_{k}^{e d^{g}}=0 \\
& A_{k} \Pi_{k}^{\ell}+B_{k} \Gamma_{k}^{\ell}-\Pi_{k}^{\ell} S_{k}^{\ell}+B_{k}^{d^{\ell}}=0 \\
& C_{k}^{e} \Pi_{k}^{\ell}+D_{k}^{e} \Gamma_{k}^{\ell}+D_{k}^{e d^{\ell}}=0 .
\end{aligned}
$$

are solvable with a solution $\Pi_{k}^{g}, \Gamma_{k}^{g}, \Pi_{k}^{\ell}, \Gamma_{k}^{\ell}$ for all $k \in \mathcal{N}$.

PROOF. We show that every solution $\Pi, \Gamma$ of (10) yields a solution $\Pi_{k}^{g}, \Gamma_{k}^{g}, \Pi_{k}^{\ell}, \Gamma_{k}^{\ell}, k \in \mathcal{N}$ of (11), (12), and vice versa. Consider (10) and partition $\Pi, \Gamma$ according to

$$
\Pi=\left[\begin{array}{cccc}
\Pi_{1}^{g} & \Pi_{11}^{\ell} & \cdots & \Pi_{1 N}^{\ell} \\
\vdots & \vdots & & \vdots \\
\Pi_{N}^{g} & \Pi_{N 1}^{\ell} & \cdots & \Pi_{N N}^{\ell}
\end{array}\right], \Gamma=\left[\begin{array}{cccc}
\Gamma_{1}^{g} & \Gamma_{11}^{\ell} & \cdots & \Gamma_{1 N}^{\ell} \\
\vdots & \vdots & & \vdots \\
\Gamma_{N}^{g} & \Gamma_{N 1}^{\dot{\ell}} & \cdots & \Gamma_{N N}^{\ell}
\end{array}\right]
$$

Using (13) and the structure of $\mathcal{A}, \mathcal{B}, \mathcal{B}_{d}$, and $\mathcal{S}$, the first equation $\mathcal{A} \Pi+\mathcal{B} \Gamma-\Pi \mathcal{S}+\mathcal{B}_{d}=0$ of (10) yields the set of matrix equations

$$
\begin{aligned}
A_{k} \Pi_{k}^{g}+B_{k} \Gamma_{k}^{g}-\Pi_{k}^{g} S^{g}+B_{k}^{d^{g}} & =0, \\
A_{k} \Pi_{k k}^{\ell}+B_{k} \Gamma_{k k}^{\ell}-\Pi_{k k}^{\ell} S_{k}^{\ell}+B_{k}^{d^{\ell}} & =0, \\
A_{k} \Pi_{k j}^{\ell}+B_{k} \Gamma_{k j}^{\ell}-\Pi_{k j}^{\ell} S_{j}^{\ell} & =0, \quad j, k \in \mathcal{N}, j \neq k .
\end{aligned}
$$

Using (13) and the structure of $\mathcal{C}_{e}, \mathcal{D}_{e}, \mathcal{D}_{e d}$, the second equation $\mathcal{C}_{e} \Pi+\mathcal{D}_{e} \Gamma+\mathcal{D}_{e d}=0$ of (10) yields the set of matrix equations

$$
\begin{aligned}
C_{k}^{e} \Pi_{k}^{g}+D_{k}^{e} \Gamma_{k}^{g}+D_{k}^{e d^{g}} & =0, \\
C_{k}^{e} \Pi_{k k}^{\ell}+D_{k}^{e} \Gamma_{k k}^{\ell}+D_{k}^{e d^{\ell}} & =0, \\
C_{k}^{e} \Pi_{k j}^{\ell}+D_{k}^{e} \Gamma_{k j}^{\ell} & =0, \quad j, k \in \mathcal{N}, j \neq k .
\end{aligned}
$$

Only If: Suppose that (13) solve (10). Then, according to (14), (17) and (15), (18), the regulator equations (11) and
(12) are solved by $\Pi_{k}^{g}, \Gamma_{k}^{g}$ and $\Pi_{k}^{\ell}=\Pi_{k k}^{\ell}, \Gamma_{k}^{\ell}=\Gamma_{k k}^{\ell}$. If: Suppose that $\Pi_{k}^{g}, \Gamma_{k}^{g}$ and $\Pi_{k k}^{\ell}, \Gamma_{k k}^{\ell}$ solve (11) and (12). It is easy to see that (16) and (19) can be satisfied by the choice $\Pi_{k j}^{\ell}=0$ and $\Gamma_{k j}^{\ell}=0$ for $j, k \in \mathcal{N}$ with $j \neq k$. Consequently, (13) with $\Pi_{k j}^{\ell}=0$ and $\Gamma_{k j}^{\ell}=0$ for $j, k \in \mathcal{N}, j \neq k$, solve the regulator equation (10).

Lemma 1 shows that due to the structure of the overall generalized plant (7) and exosystem (8), the existence of a solution to (10) is equivalent to the existence of solutions to the local regulator equations (11), (12). Consequently, the solvability of (11), (12) is a necessary condition, not only for the existence of a distributed regulator but also for the existence of a centralized regulator with global information. The following assumptions will be needed in the sequel.

Assumption 1 The graph $\mathcal{G}$ is connected and, without loss of generality, node 1 is the root of a spanning tree.

Assumption 2 For all $k \in \mathcal{N},\left(A_{k}, B_{k}\right)$ is stabilizable.

Assumption 3 All of the following pairs are detectable:

$$
\begin{aligned}
& \left(\left[\begin{array}{ccc}
A_{1} & B_{1}^{d^{g}} & B_{1}^{d^{\ell}} \\
0 & S^{g} & 0 \\
0 & 0 & S_{1}^{\ell}
\end{array}\right],\left[\begin{array}{lll}
C_{1} & D_{1}^{d^{g}} & D_{1}^{d^{\ell}}
\end{array}\right]\right) \\
& \left(\left[\begin{array}{cc}
A_{k} & B_{k}^{d^{\ell}} \\
0 & S_{k}^{\ell}
\end{array}\right],\left[\begin{array}{ll}
C_{k} & D_{k}^{d^{\ell}}
\end{array}\right]\right), \quad k \in \mathcal{N} \backslash\{1\}
\end{aligned}
$$

Assumption 4 The local regulator equations (11) and (12) are solvable with a solution $\Pi_{k}^{g}, \Gamma_{k}^{g}, \Pi_{k}^{\ell}, \Gamma_{k}^{\ell}$ for all $k \in \mathcal{N}$.

In the following, we briefly discuss each assumption separately. Assumption 1 is required for the construction of a distributed regulator, cf., [26]. Assumption 2 is equivalent to stabilizability of the pair $(\mathcal{A}, \mathcal{B})$ due to the structure of (7). It is required for P1) since (1a) may be unstable. Assumption 3 ensures detectability of (9) due to the structure of (7), (8). The pairs (21) are necessarily detectable if the pair (9) is. The detectability of (20) allows agent 1 to reconstruct $d^{g}$. Assumption 4 is motivated by Lemma 1 . It is a necessary condition for the existence of a distributed (as well as a centralized) regulator solving Problem 1. Hence it causes no loss of generality.

\section{The Distributed Regulator}

The following result is the general solution to Problem 1. In its original form, the distributed regulator is due to $\mathrm{Su}$ and Huang [26]. Here we present a refined formulation taking into account both global and local exosystems.

Theorem 3 Consider a group of agents (1) with exosystems (2), (3). Suppose that Assumptions 1-4 are satisfied. Then, a distributed regulator solving Problem 1 can be constructed as follows: 
- For all $k \in \mathcal{N}$, choose $F_{k}$ such that $A_{k}-B_{k} F_{k}$ is Hurwitz.

- For all $k \in \mathcal{N}$, find a solution for (11) and (12) and set

$$
G_{k}^{g}=\Gamma_{k}^{g}+F_{k} \Pi_{k}^{g}, \quad G_{k}^{\ell}=\Gamma_{k}^{\ell}+F_{k} \Pi_{k}^{\ell} .
$$

- Choose observer gain matrices $L_{1}$ for (20) and $L_{k}, k \in$ $\mathcal{N} \backslash\{1\}$, for (21) and construct the local observers

$$
\left[\begin{array}{c}
\hat{x}_{1} \\
\dot{d}_{1}^{g} \\
\dot{\hat{d}}_{1}^{\ell}
\end{array}\right]=\left[\begin{array}{ccc}
A_{1} & B_{1}^{d^{g}} & B_{1}^{d^{\ell}} \\
0 & S^{g} & 0 \\
0 & 0 & S_{1}^{\ell}
\end{array}\right]\left[\begin{array}{c}
\hat{x}_{1} \\
\hat{d}_{1}^{g} \\
\hat{d}_{1}^{\ell}
\end{array}\right]+\left[\begin{array}{c}
B_{1} \\
0 \\
0
\end{array}\right] u_{1}+L_{1}\left(y_{1}-\hat{y}_{1}\right)
$$$$
\left[\begin{array}{c}
\dot{\hat{x}}_{k} \\
\hat{\hat{d}}_{k}^{\ell}
\end{array}\right]=\left[\begin{array}{cc}
A_{k} & B_{k}^{d^{\ell}} \\
0 & S_{k}^{\ell}
\end{array}\right]\left[\begin{array}{c}
\hat{x}_{k} \\
\hat{d}_{k}^{\ell}
\end{array}\right]+\left[\begin{array}{cc}
B_{k} & B_{k}^{d^{g}} \\
0 & 0
\end{array}\right]\left[\begin{array}{c}
u_{k} \\
\hat{d}_{k}^{g}
\end{array}\right]+L_{k}\left(y_{k}-\hat{y}_{k}\right),
$$

where $\hat{y}_{k}=C_{k} \hat{x}_{k}+D_{k}^{d^{g}} \hat{d}_{k}^{g}+D_{k}^{d^{\ell}} \hat{d}_{k}^{\ell}+D_{k} u_{k}$ for all $k \in \mathcal{N}$.

- Let $\mathcal{G}_{1}$ be the graph $\mathcal{G}$ after deletion of all incoming edges to node 1. Choose $K$ such that $S^{g}-\lambda_{k} K$ is Hurwitz for the non-zero eigenvalues $\lambda_{k}, k=2, \ldots, N$, of the Laplacian matrix $L_{\mathcal{G}_{1}}$ and set for all $k \in \mathcal{N} \backslash\{1\}$,

$$
\dot{\hat{d}}_{k}^{g}=S^{g} \hat{d}_{k}^{g}+K \sum_{j \in \mathcal{N}_{k}}\left(\hat{d}_{j}^{g}-\hat{d}_{k}^{g}\right)
$$

Finally, the control law for each agent $k \in \mathcal{N}$ is given by

$$
u_{k}=-F_{k} \hat{x}_{k}+G_{k}^{g} \hat{d}_{k}^{g}+G_{k}^{\ell} \hat{d}_{k}^{\ell} .
$$

PROOF. For each $k \in \mathcal{N}$, we define the observer errors

$$
\varepsilon_{k}^{x}=x_{k}-\hat{x}_{k}, \quad \varepsilon_{k}^{d^{\ell}}=d_{k}^{\ell}-\hat{d}_{k}^{\ell}, \quad \varepsilon_{k}^{d^{g}}=d^{g}-\hat{d}_{k}^{g} .
$$

System (23) is a Luenberger observer and it is immediate that $\varepsilon_{1}^{x}(t), \varepsilon_{1}^{d^{\ell}}(t), \varepsilon_{1}^{d^{g}}(t)$ converge to zero as $t \rightarrow \infty$. Node 1 is the only root of a spanning tree in $\mathcal{G}_{1}$. Substituting $\hat{d}_{1}^{g}=d^{g}-\varepsilon_{1}^{d^{g}}$ in (25) reveals that $(2),(25)$ form a leader-follower network with leader (2) corresponding to node 1 . Since $\varepsilon_{1}^{d^{g}}(t) \rightarrow 0$, it follows that $\hat{d}_{k}^{g}(t)-d^{g}(t) \rightarrow 0$ as $t \rightarrow \infty$, cf., [31]. A suitable gain matrix $K$ in (25) exists by [31, Thm. 3.3] and a design method is given in Theorem 1. For all $k \in \mathcal{N} \backslash\{1\}$, we have

$$
\begin{gathered}
{\left[\begin{array}{c}
\dot{\varepsilon}_{k}^{x} \\
\dot{\varepsilon}_{k}^{d^{\ell}}
\end{array}\right]=\left(\left[\begin{array}{cc}
A_{k} & B_{k}^{d^{\ell}} \\
0 & S_{k}^{\ell}
\end{array}\right]-L_{k}\left[\begin{array}{ll}
C_{k} & D_{k}^{d^{\ell}}
\end{array}\right]\right)\left[\begin{array}{c}
\varepsilon_{k}^{x} \\
\varepsilon_{k}^{d^{\ell}}
\end{array}\right]} \\
+\left(\left[\begin{array}{c}
B_{k}^{d^{g}} \\
0
\end{array}\right]-L_{k} D_{k}^{d^{g}}\right) \varepsilon_{k}^{d^{g}} .
\end{gathered}
$$

It follows that $\varepsilon_{k}^{x}(t) \rightarrow 0, \varepsilon_{k}^{d^{\ell}}(t) \rightarrow 0$ as $t \rightarrow \infty$ for all $k \in \mathcal{N}$, since the error system matrices are Hurwitz by construction. Next, we define the transient state component of agent $k$ as

$$
\varepsilon_{k}=x_{k}-\Pi_{k}^{g} d^{g}-\Pi_{k}^{\ell} d_{k}^{\ell} .
$$

It is the state component in the complement of the subspace $\mathcal{V}_{k}^{+}=\left\{\left(x_{k}, d^{g}, d_{k}^{\ell}\right): x_{k}=\Pi_{k}^{g} d^{g}+\Pi_{k}^{\ell} d_{k}^{\ell}\right\}$. As discussed in [13], the equations (11a), (12a) express the fact that the subspace $\mathcal{V}_{k}^{+}$is a controlled invariant subspace of the system

$$
\left[\begin{array}{c}
\dot{x}_{k} \\
\dot{d}^{g} \\
\dot{d}_{k}^{\ell}
\end{array}\right]=\left[\begin{array}{ccc}
A_{k} & B_{k}^{d^{g}} & B_{k}^{d^{\ell}} \\
0 & S^{g} & 0 \\
0 & 0 & S_{k}^{\ell}
\end{array}\right]\left[\begin{array}{c}
x_{k} \\
d^{g} \\
d_{k}^{\ell}
\end{array}\right]+\left[\begin{array}{c}
B_{k} \\
0 \\
0
\end{array}\right] u_{k}
$$

and $\mathcal{V}_{k}^{+}$is rendered invariant by $u_{k}=\Gamma_{k}^{g} d^{g}+\Gamma_{k}^{\ell} d_{k}^{\ell}$. Moreover, this subspace lies in the null space of the regulation error map (1c) due to (11b), (12b). As shown next, $\mathcal{V}_{k}^{+}$is rendered asymptotically stable by the proposed distributed regulator, and $e_{k}(t)$ indeed converges to zero for all initial conditions. Using (1a), (2), (3), (22), (26), (27), and (28), we compute

$$
\begin{aligned}
\dot{\varepsilon}_{k}= & \dot{x}_{k}-\Pi_{k}^{g} \dot{d}^{g}-\Pi_{k}^{\ell} \dot{d}_{k}^{\ell} \\
= & \left(A_{k}-B_{k} F_{k}\right) \varepsilon_{k}+B_{k} F_{k} \varepsilon_{k}^{x}-B_{k} G_{k}^{g} \varepsilon_{k}^{d^{g}}-B_{k} G_{k}^{\ell} \varepsilon_{k}^{d^{\ell}} \\
& +\left(A_{k} \Pi_{k}^{g}+B_{k} \Gamma_{k}^{g}-\Pi_{k}^{g} S^{g}+B_{k}^{d^{g}}\right) d^{g} \\
& +\left(A_{k} \Pi_{k}^{\ell}+B_{k} \Gamma_{k}^{\ell}-\Pi_{k}^{\ell} S_{k}^{\ell}+B_{k}^{d^{\ell}}\right) d_{k}^{\ell} .
\end{aligned}
$$

With (11a) and (12a), this reduces to

$$
\dot{\varepsilon}_{k}=\left(A_{k}-B_{k} F_{k}\right) \varepsilon_{k}+B_{k} F_{k} \varepsilon_{k}^{x}-B_{k} G_{k}^{g} \varepsilon_{k}^{d^{g}}-B_{k} G_{k}^{\ell} \varepsilon_{k}^{d^{\ell}} .
$$

Since the observer errors $\varepsilon_{k}^{x}, \varepsilon_{k}^{d^{g}}, \varepsilon_{k}^{d^{\ell}}$ converge to zero and $A_{k}-B_{k} F_{k}$ is Hurwitz, it follows that $\varepsilon_{k}(t) \rightarrow 0$ as $t \rightarrow \infty$ for all $k \in \mathcal{N}$. If $d^{g}(0)=0$ and $d_{k}^{\ell}(0)=0$ for all $k \in \mathcal{N}$, then $x_{k}(t)=\varepsilon_{k}(t) \rightarrow 0$ as $t \rightarrow \infty$, and since the observer errors converge to zero, also $\hat{x}_{k}(t) \rightarrow 0, \hat{d}_{k}^{g}(t) \rightarrow 0$, and $\hat{d}_{k}^{\ell}(t) \rightarrow 0$ as $t \rightarrow \infty$. Consequently, P1) is fulfilled. It remains to show that the regulation errors $e_{k}$ converge to zero for all initial conditions. Using (1c), (22), (26), and (27), we compute

$$
\begin{aligned}
e_{k}= & \left(C_{k}^{e}-D_{k}^{e} F_{k}\right) \varepsilon_{k}-D_{k}^{e}\left(-F_{k} \varepsilon_{k}^{x}+G_{k}^{g} \varepsilon_{k}^{d^{g}}+G_{k}^{\ell} \varepsilon_{k}^{d^{\ell}}\right) \\
& +\left(C_{k}^{e} \Pi_{k}^{g}+D_{k}^{e} \Gamma_{k}^{g}+D_{k}^{e d^{g}}\right) d^{g}+\left(C_{k}^{e} \Pi_{k}^{\ell}+D_{k}^{e} \Gamma_{k}^{\ell}+D_{k}^{e d^{\ell}}\right) d_{k}^{\ell} .
\end{aligned}
$$

With (11b) and (12b), this reduces to

$e_{k}=\left(C_{k}^{e}-D_{k}^{e} F_{k}\right) \varepsilon_{k}-D_{k}^{e}\left(-F_{k} \varepsilon_{k}^{x}+G_{k}^{g} \varepsilon_{k}^{d^{g}}+G_{k}^{\ell} \varepsilon_{k}^{d^{\ell}}\right)$.

Both $\varepsilon_{k}$ and observer errors $\varepsilon_{k}^{x}, \varepsilon_{k}^{d^{g}}, \varepsilon_{k}^{d^{\ell}}$ converge to zero. Hence, $e_{k}(t) \rightarrow 0$ as $t \rightarrow \infty$ for all $k \in \mathcal{N}$, P2) is fulfilled.

\section{Transient Synchronization: Identical Agents}

The distributed regulator presented in Theorem 3 solves Problem 1 and hence realizes a cooperative behavior of the group. In this section, we show that the cooperative behavior can be improved significantly by a suitable extension of the distributed regulator. As motivated in the introduction, we enable a cooperative reaction on local disturbances, meaning that a suitably defined synchronization error is kept small in transient phases. We propose a novel distributed regulator with additional couplings among the agents which stabilize the synchronization error and guarantee a desired performance. In a first step, we consider groups of agents with identical dynamics. 
Assumption 5 There exist matrices $A, B, C^{e}, D^{e}, F$ such that for all $k \in \mathcal{N}, A_{k}=A, B_{k}=B, C_{k}^{e}=C^{e}, D_{k}^{e}=D^{e}, F_{k}=F$.

Assumption 5 does not require all matrices in (1) to be identical for the individual agents. The measurement output maps (1b) are allowed to be non-identical, and the local exosystems (3) as well as the generalized disturbance input matrices in (1a), (1c), can be non-identical. This leaves great flexibility in the problem formulation. Moreover, Assumption 5 will be relaxed in Section 5 .

The disagreement of the group can be quantified based on the transient state components $\varepsilon_{k}$ defined in (28) since, according to (30), $e_{k}=\left(C^{e}-D^{e} F\right) \varepsilon_{k}$ under Assumption 5 and without observer errors. Agreement of $\varepsilon_{k}$ implies agreement of the regulation errors $e_{k}$. For each agent $k$, we define the transient synchronization error

$$
\varepsilon_{k}^{s}=\varepsilon_{k}-\frac{1}{N} \sum_{j=1}^{N} \varepsilon_{j}
$$

In the following, we propose a distributed regulator which solves Problem 1 and, additionally, exponentially stabilizes the synchronization errors $\varepsilon_{k}^{s}$ with a certain desired decay rate $\gamma>0$. In order to guarantee that this decay rate can be achieved, we refine Assumptions 2 and 3 as follows.

Assumption 2' For a given $\gamma>0$, the pairs $\left(A_{k}, B_{k}\right), k \in \mathcal{N}$, are stabilizable with decay rate $\gamma$.

Assumption 3' For a given $\eta>0$, the pairs (20) and (21) are detectable with decay rate $\eta$.

Theorem 4 Consider a group of agents (1) with exosystems (2), (3). Suppose that Assumptions 1, 2', 3', 4, and 5 are satisfied for some $\eta>\gamma>0$. Then, a distributed regulator solving Problem 1 and additionally achieving exponential stability of synchronization errors $\varepsilon_{k}^{s}$ with decay rate $\gamma>0$ can be constructed as follows:

- Choose F such that A-BF is Hurwitz.

- For all $k \in \mathcal{N}$, find a solution for (11) and (12) and set $G_{k}^{g}=\Gamma_{k}^{g}+F \Pi_{k}^{g}, G_{k}^{\ell}=\Gamma_{k}^{\ell}+F \Pi_{k}^{\ell}$.

- Choose $L_{k}$ and $K$ in (23), (24), (25) such that the corresponding error dynamics are exponentially stable with decay rate $\eta>0$.

- Choose $H$ such that for all non-zero eigenvalues $\lambda_{k}, k=$ $2, \ldots, N$, of the Laplacian matrix $L_{\mathcal{G}}$,

$$
\max \left\{\operatorname{Re}(\mu): \mu \in \sigma\left(A-B F-\lambda_{k} B H\right)\right\}<-\gamma .
$$

Finally, the control law for each agent $k \in \mathcal{N}$ is given by

$$
u_{k}=-F \hat{x}_{k}+G_{k}^{g} \hat{d}_{k}^{g}+G_{k}^{\ell} \hat{d}_{k}^{\ell}+H \sum_{j \in \mathcal{N}_{k}}\left(\hat{\varepsilon}_{j}-\hat{\varepsilon}_{k}\right)
$$

where $\hat{\varepsilon}_{k}=\hat{x}_{k}-\Pi_{k}^{g} \hat{d}_{k}^{g}-\Pi_{k}^{\ell} \hat{d}_{k}^{\ell}$.

PROOF. Firstly, we consider the full-information regulator

$$
u_{k}=-F x_{k}+G_{k}^{g} d^{g}+G_{k}^{\ell} d_{k}^{\ell}+H \sum_{j \in \mathcal{N}_{k}}\left(\varepsilon_{j}-\varepsilon_{k}\right) .
$$

As in (29), the dynamics of $\varepsilon_{k}$ can be computed as

$$
\dot{\varepsilon}_{k}=(A-B F) \varepsilon_{k}+B H \sum_{j \in \mathcal{N}_{k}}\left(\varepsilon_{j}-\varepsilon_{k}\right) .
$$

The novel term in the control law (34) couples the transient state components $\varepsilon_{k}$ of the agents. Eq. (35) is a diffusively coupled network of $N$ identical stable linear systems. We apply the transformation $\tilde{\varepsilon}=\left(T^{-1} \otimes I_{n^{x}}\right) \varepsilon$ with $T$ such that $\Lambda=T^{-1} L_{\mathcal{G}} T$ is upper-triangular, the first column of $T$ is $\mathbf{1}$, and the first row of $T^{-1}$ is $p^{\top}$, with $p^{\top} L_{\mathcal{G}}=0^{\top}, p^{\top} \mathbf{1}=1$, cf., [5]. This leads to $\dot{\tilde{\varepsilon}}=\left(I_{N} \otimes(A-B F)-\Lambda \otimes(B H)\right) \tilde{\varepsilon}$. Let $\tilde{\varepsilon}$ be partitioned into $\tilde{\varepsilon}^{\prime}$ and $\tilde{\varepsilon}^{\prime \prime}$. Then, $\dot{\tilde{\varepsilon}}^{\prime}=(A-B F) \tilde{\varepsilon}^{\prime}$ and

$$
\dot{\tilde{\varepsilon}}^{\prime \prime}=\left[\begin{array}{ccc}
A-B F-\lambda_{2} B H & \cdots & \star \\
0 & \ddots & \vdots \\
& & A-B F-\lambda_{N} B H
\end{array}\right] \tilde{\varepsilon}^{\prime \prime} .
$$

By (32), the maximal real part of the eigenvalues of the latter matrix is smaller than $-\gamma$. Such a gain $H$ exists by Assumptions 2' and [31, Thm. 3.3]. Hence, there exists a constant $\tilde{c}>0$ such that $\left\|\tilde{\varepsilon}^{\prime \prime}(t)\right\| \leq \tilde{c}\left\|\tilde{\varepsilon}^{\prime \prime}(0)\right\| e^{-\gamma t}$, for all $t \geq 0$, cf., [3]. We define the projection matrix $P=I_{N}-\frac{1}{N} \mathbf{1 1}^{\top}$ and partition $T=\left[\begin{array}{ll}1 & T^{\prime \prime}\end{array}\right]$. The stack vector of synchronization errors (31) is given by $\varepsilon^{s}=\left(P \otimes I_{n^{x}}\right) \varepsilon=\left(P T \otimes I_{n^{x}}\right) \tilde{\varepsilon}=\left(P T^{\prime \prime} \otimes I_{n^{x}}\right) \tilde{\varepsilon}^{\prime \prime}$ since $P \mathbf{1}=0$. Hence, it follows that there exists a constant $c>0$ such that $\left\|\varepsilon^{s}(t)\right\| \leq c\left\|\left(P \otimes I_{n^{x}}\right) \varepsilon(0)\right\| e^{-\gamma t}$. Consequently, the synchronization error is exponentially stable with a decay rate of at least $\gamma$. The matrix $A-B F$ is Hurwitz by the choice of $F$, which guarantees that $\tilde{\varepsilon}^{\prime}(t) \rightarrow 0$ as $t \rightarrow \infty$. Therefore, it holds that $\varepsilon(t)=\left(T \otimes I_{n^{x}}\right) \tilde{\varepsilon}(t) \rightarrow 0$ as $t \rightarrow \infty$. Since $e_{k}=\left(C^{e}-D^{e} F\right) \varepsilon_{k}$, Problem 1 is solved.

Secondly, we focus on the output feedback regulator (33). Note that $\hat{\varepsilon}_{k}=\hat{x}_{k}-\Pi_{k}^{g} \hat{d}_{k}^{g}-\Pi_{k}^{\ell} \hat{d}_{k}^{\ell}=\varepsilon_{k}-\varepsilon_{k}^{x}+\Pi_{k}^{g} \varepsilon_{k}^{d^{g}}+\Pi_{k}^{\ell} \varepsilon_{k}^{d^{\ell}}$. The dynamics of the transient state components can be computed as $\dot{\varepsilon}_{k}=(A-B F) \varepsilon_{k}+B H \sum_{j \in \mathcal{N}_{k}}\left(\varepsilon_{j}-\varepsilon_{k}\right)+\delta_{k}(t)$, where $\delta_{k}(t)$ captures the influence of the observer errors, i.e., $\delta_{k}(t)=B F \varepsilon_{k}^{x}-B G_{k}^{g} \varepsilon_{k}^{d^{g}}-B G_{k}^{\ell} \varepsilon_{k}^{d^{\ell}}-B H \sum_{j \in \mathcal{N}_{k}}\left(\varepsilon_{j}^{x}-\right.$ $\left.\Pi_{j}^{g} \varepsilon_{j}^{d^{g}}-\Pi_{j}^{\ell} \varepsilon_{j}^{d^{\ell}}-\varepsilon_{k}^{x}+\Pi_{k}^{g} \varepsilon_{k}^{d^{g}}+\Pi_{k}^{\ell} \varepsilon_{k}^{d^{\ell}}\right)$. The novel term in (33) has no influence on the dynamics of the observer errors $\varepsilon_{k}^{x}, \varepsilon_{k}^{d^{\ell}}, \varepsilon_{k}^{d^{g}}$. They obey the same dynamics as in the proof of Theorem 3. Moreover, by assumption, they converge to zero exponentially with decay rate $\eta$. Let $\delta=\left[\begin{array}{lll}\delta_{1}^{\top} & \cdots & \delta_{N}^{\top}\end{array}\right]^{\top}$. It follows that there exists a constant $c_{\delta}$ such that $\|\delta(t)\| \leq c_{\delta}\|\delta(0)\| e^{-\eta t}$ for all $t \geq 0$. With the state transformation as before, this leads to $\dot{\tilde{\varepsilon}}=\left(I_{N} \otimes(A-B F)-\Lambda \otimes B H\right) \tilde{\varepsilon}+\left(T^{-1} \otimes I_{n^{x}}\right) \delta$. Since $\eta>\gamma$, it follows that $\tilde{\varepsilon}^{\prime \prime}(t) \rightarrow 0$ exponentially as $t \rightarrow \infty$ with decay rate $\gamma$. Moreover, we also have $\tilde{\varepsilon}^{\prime}(t) \rightarrow 0$ as $t \rightarrow \infty$. Since $\varepsilon_{k}(t) \rightarrow 0$ as $t \rightarrow \infty$ for all $k \in \mathcal{N}$, the coupling term in (33) vanishes asymptotically. Consequently, analogously to the proof of Theorem 3, it holds that $e_{k}(t) \rightarrow 0$ as $t \rightarrow \infty$ and Problem 1 is solved.

The novel control law (33) solves Problem 1 and additionally enforces synchronization of the regulation errors with a 
desired decay rate and therefore has the desired effect, that is, a cooperative reaction of the group on disturbances acting on individual agents. The gain matrices $F$ and $H$ allow to tune separately the local disturbance rejection of each agent and the synchronization of the group. Theorem 1 serves as a design method for $H$.

Example 1 We consider a group of $N=5$ vehicles in a platoon, each modeled as a double-integrator system of the form $\ddot{s}_{k}=u_{k}+w_{k}$, where $s_{k}$ is the vehicle position, $\dot{s}_{k}=v_{k}$ its velocity, $u_{k}$ its control input, and $w_{k}$ a local disturbance acting on vehicle $k$, e.g., due to a mass change, gear shift, or other external influence. There is a ramp shaped global reference signal $s_{0}(t), \dot{s}_{0}(t)=v_{0}$, for the position of the first vehicle. Moreover, there are constant local reference signals $r_{k}$ dictating the relative position of the $k$-th vehicle to the global reference. This scenario is captured by

$$
\begin{aligned}
\dot{x}_{k} & =\left[\begin{array}{ll}
0 & 1 \\
0 & 0
\end{array}\right] x_{k}+\left[\begin{array}{l}
0 \\
1
\end{array}\right] u_{k}+\left[\begin{array}{ll}
0 & 0 \\
0 & 0
\end{array}\right] d^{g}+\left[\begin{array}{ll}
0 & 0 \\
0 & 1
\end{array}\right] d_{k}^{\ell} \\
y_{k} & =\left[\begin{array}{ll}
0 & 0 \\
1 & 0 \\
0 & 1
\end{array}\right] x_{k}+\left[\begin{array}{ll}
0 & 0 \\
0 & 0 \\
0 & 0
\end{array}\right] u_{k}+\left[\begin{array}{ll}
0 & 0 \\
0 & 0 \\
0 & 0
\end{array}\right] d^{g}+\left[\begin{array}{ll}
1 & 0 \\
0 & 0 \\
0
\end{array}\right] d_{k}^{\ell} \\
e_{k} & =\left[\begin{array}{ll}
1 & 0 \\
0 & 1
\end{array}\right] x_{k}+\left[\begin{array}{ll}
0 & 0 \\
0 & 0
\end{array}\right] u_{k}+\left[\begin{array}{cc}
-1 & 0 \\
0 & -1
\end{array}\right] d^{g}+\left[\begin{array}{ll}
0 & -1 \\
0 & 0
\end{array}\right] d_{k}^{\ell}
\end{aligned}
$$

with $x_{k}=\left[\begin{array}{ll}s_{k} & v_{k}\end{array}\right]^{\top}$ for all $k \in \mathcal{N}$. Only for vehicle 1 , we have

$$
y_{1}=\left[\begin{array}{ll}
0 & 0 \\
0 & 0 \\
0 & 0 \\
1 & 0 \\
0 & 1
\end{array}\right] x_{1}+\left[\begin{array}{ll}
0 & 0 \\
0 & 0 \\
0 & 0 \\
0 & 0 \\
0 & 0
\end{array}\right] u_{1}+\left[\begin{array}{ll}
1 & 0 \\
0 & 1 \\
0 & 0 \\
0 & 0 \\
0 & 0
\end{array}\right] d^{g}+\left[\begin{array}{ll}
0 & 0 \\
0 & 0 \\
1 & 0 \\
0 & 0 \\
0 & 0
\end{array}\right] d_{1}^{\ell} \text {. }
$$

The generalized disturbances $d^{g}=\left[\begin{array}{ll}s_{0} & v_{0}\end{array}\right]^{\top}$ and $d_{k}^{\ell}=$ $\left[\begin{array}{ll}r_{k} & w_{k}\end{array}\right]^{\top}$ are generated by $\dot{d}^{g}=\left[\begin{array}{ll}0 & 1 \\ 0 & 0\end{array}\right] d^{g}$ and $\dot{d}_{k}^{\ell}=\left[\begin{array}{ll}0 & 0 \\ 0 & 0\end{array}\right] d_{k}^{\ell}$, respectively. Each vehicle is assumed to communicate with its follower and predecessor in the string, i.e., $\mathcal{G}$ is an undirected line graph. All assumptions of Theorem 4 are satisfied and a distributed output feedback regulator can be constructed as follows. We find $F_{k}=[0.70711 .3836]$ via LQR design. Equations (11), (12) are solved by $\Pi_{k}^{g}=\left[\begin{array}{ll}1 & 0 \\ 0 & 1\end{array}\right], \Gamma_{k}^{g}=\left[\begin{array}{ll}0 & 0\end{array}\right]$, $\Pi_{k}^{\ell}=\left[\begin{array}{ll}1 & 0 \\ 0 & 0\end{array}\right], \Gamma_{k}^{\ell}=\left[\begin{array}{ll}0-1\end{array}\right]$, which yields $G_{k}^{g}=\Gamma_{k}^{g}+F_{k} \Pi_{k}^{g}$ and $G_{k}^{\ell}=\Gamma_{k}^{\ell}+F_{k} \Pi_{k}^{\ell}$. We construct local observers (23), (24) so that the observer errors are exponentially stable with decay rate 3. The corresponding observer gain matrices $L_{1}, L_{k}, k \in \mathcal{N} \backslash\{1\}$ are found by $L Q R$ design exploiting duality. The coupling gains $K=\left[\begin{array}{ll}-20.9938 & -1.6601 \\ -0.0261 & -21.0682\end{array}\right]$ and $H=[-14.6573-7.0411]$ are designed via Theorem 1 for $\gamma=2$ and $\eta=2.5$ with LMI regions $S(2,25, \pi / 4)$ and $S(2.5,75, \pi / 4)$, respectively, $c f .,[24]$.

In the simulations, we set $s_{0}(0)=20, v_{0}=2$, and $r_{k}=$ $-5(k-1)$. In order to assess the behavior of the closed loop, particularly in transient phases, the step signals $w_{1}(t)=$ -100 for $t \geq t_{1}, w_{2}(t)=-100$ for $t \geq t_{2}, w_{3}(t)=-100$ for $t \geq t_{3}, w_{4}(t)=-100$ for $t \geq t_{4}$ are applied to the vehicles. The distributed regulator (26) without the additional coupling term $\left(H=\left[\begin{array}{ll}0 & 0\end{array}\right]\right)$ leads to the simulation result shown in Fig. 1(a). The distributed regulation problem is solved, i.e., the reference signals are tracked and the disturbances are rejected asymptotically. Nevertheless, it becomes apparent that the group does not react cooperatively on local disturbances. The step disturbances are rejected by the local

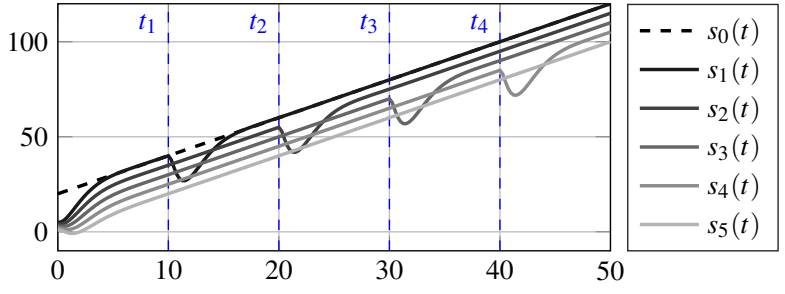

(a) Simulation result for the distributed regulator in Theorem 3 .

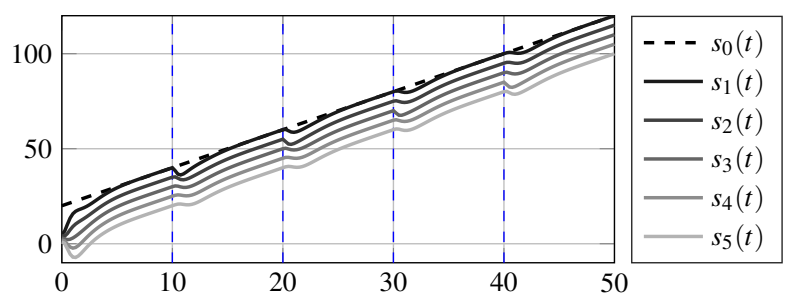

(b) Simulation result for the distributed regulator in Theorem 4.

Fig. 1. Simulation results for the vehicle platoon (Example 1).

controllers of the respective agents, but the other agents in the group have no information about the disturbance and cannot adjust their actions. A more desirable reaction is that the other vehicles in the platoon slow down or accelerate in order to maintain the desired formation during the transient phases since maintaining the desired inter-vehicle distances is of higher importance than maintaining the desired velocity for a vehicle platoon. The novel distributed regulator (33) takes this requirement into account. The corresponding simulation result in Fig. 1(b) shows that the effect of the disturbances on the inter-vehicle distances is indeed rejected much more efficiently. The vehicles react cooperatively and maintain a small synchronization error. The Multi-Parametric Toolbox [7] and Yalmip [16] were used for the simulations.

\section{Transient Synchronization: Non-identical Agents}

In this section, we aim at relaxing Assumption 5. In case of non-identical agents, an analogue coupling term as in (34) leads to a diffusively coupled network of non-identical stable linear systems in (35). In this case, it is hard to find a suitable coupling gain $H$. In case of mismatching state dimensions $n_{k}^{x}$ such couplings cannot be realized. We exclude this case and consider heterogeneous networks consisting of non-identical perturbed versions of a common nominal agent. We propose to design the couplings for the nominal dynamics and evaluate the design for the heterogeneous network a posteriori. Moreover, we show how to compute the size of tolerable stable additive perturbations in terms of their $\mathcal{H}_{\infty}$ norm, which yields a stability guarantee for a whole class of non-identical agents. For ease of presentation, we discuss only the full information case in the following. The output feedback implementation can be carried out analogously to Theorem 4. 
Suppose that we implement a control law like (33) in a group of non-identical agents, i.e.,

$$
u_{k}=-F_{k} x_{k}+G_{k}^{g} d^{g}+G_{k}^{\ell} d_{k}^{\ell}+H \sum_{j \in \mathcal{N}_{k}}\left(\varepsilon_{j}-\varepsilon_{k}\right)
$$

where $F_{k}, G_{k}^{g}, G_{k}^{\ell}$ are designed as in Theorem 3 and $H$ is a coupling gain to be specified in the following. Then, analogously to (35), the error variables $\varepsilon_{k}$ obey the dynamics

$$
\dot{\varepsilon}_{k}=\left(A_{k}-B_{k} F_{k}\right) \varepsilon_{k}+B_{k} v_{k},
$$

where $A_{k}-B_{k} F_{k}$ is Hurwitz and $v_{k}=H \sum_{j \in \mathcal{N}_{k}}\left(\varepsilon_{j}-\varepsilon_{k}\right)$. The key assumption in the following is that the systems (37) have similar dynamics.

Design of the couplings for nominal dynamics: The following procedure may be used in order to design $H$ in (36). First, define a nominal system $\dot{z}_{k}=\tilde{A} z_{k}+\tilde{B} v_{k}$ for (37), where $\tilde{A}$ is Hurwitz. We use the average matrices $\tilde{A}=\frac{1}{N} \sum_{k=1}^{N}\left(A_{k}-B_{k} F_{k}\right), \tilde{B}=\frac{1}{N} \sum_{k=1}^{N} B_{k}$. Alternatively, if this does not yield $\tilde{A}$ Hurwitz, one can choose a particular agent $j \in \mathcal{N}$ as the nominal system by setting $\tilde{A}=A_{j}-B_{j} F_{j}$, $\tilde{B}=B_{j}$. Second, design the coupling gain $H$ for the nominal system such that for all $k \in \mathcal{N} \backslash\{1\}, \max \{\operatorname{Re}(\mu): \mu \in$ $\left.\sigma\left(\tilde{A}-\lambda_{k} \tilde{B} H\right)\right\}<-\gamma$, using Theorem 1. Third, implement (36) in the group of non-identical agents and evaluate the design. In particular, it has to be checked whether the network (37) with $v_{k}=H \sum_{j \in \mathcal{N}_{k}}\left(\varepsilon_{j}-\varepsilon_{k}\right), k \in \mathcal{N}$, is asymptotically stable and has a satisfactory performance.

Robustness analysis: Besides evaluating the design for a particular set of non-identical agents, it is possible to quantify the tolerable heterogeneity in the agent dynamics for a given coupling gain $H$ via a robustness analysis as follows. The nominal transfer function matrix from $v_{k}$ to $z_{k}$ is given by

$$
G(s)=\left(s I_{n^{x}}-\tilde{A}\right)^{-1} \tilde{B} .
$$

The transfer function matrix from $v_{k}$ to $\varepsilon_{k}$ is $G_{k}(s)=\left(s I_{n^{x}}-\right.$ $\left.\left(A_{k}-B_{k} F_{k}\right)\right)^{-1} B_{k}$, for $k \in \mathcal{N}$. Then, $G_{k}(s)$ can be expressed as perturbed version of the nominal $G$ with an additive uncertainty $\Delta_{k}(s)=G_{k}(s)-G(s)$. Disconnecting $\Delta_{k}$ leads to the description

$$
\left[\begin{array}{l}
\zeta_{k} \\
\varepsilon_{k}
\end{array}\right]=\left[\begin{array}{cc}
0 & I_{n^{x}} \\
I_{n^{x}} & G
\end{array}\right]\left[\begin{array}{l}
\omega_{k} \\
v_{k}
\end{array}\right], \quad \omega_{k}=\Delta_{k} \zeta_{k} .
$$

Lemma 2 Let the graph $\mathcal{G}$ be undirected and connected. Suppose that $G_{k}(s)$ can be expressed as $G_{k}(s)=G(s)+\Delta_{k}(s)$ for some real-rational strictly proper stable $\Delta_{k}(s), k \in \mathcal{N}$. Suppose that there exist a scalar $\eta>0$ and positive definite matrices $X_{k}>0$ such that

$$
\left[\begin{array}{ccc}
\left(\tilde{A}-\lambda_{k} \tilde{B} H\right)^{\top} X_{k}+X_{k}\left(\tilde{A}-\lambda_{k} \tilde{B} H\right) & -\lambda_{k} X_{k} \tilde{B} H & -\lambda_{k} H^{\top} \\
-\lambda_{k} H^{\top} \tilde{B}^{\top} X_{k} & -\eta I_{n^{x}} & -\lambda_{k} H^{\top} \\
-\lambda_{k} H & -\lambda_{k} H & -\eta I_{n^{u}}
\end{array}\right]<0
$$

for $k=2, \ldots, N$ and $\left\|\Delta_{k}\right\|_{\infty}<1 / \eta$ for all $k \in \mathcal{N}$. Then, the network (37) with $v_{k}=H \sum_{j \in \mathcal{N}_{k}}\left(\varepsilon_{j}-\varepsilon_{k}\right), k \in \mathcal{N}$, is asymptotically stable.
PROOF. A state-space description of (39) is given by $\dot{z}_{k}=\tilde{A} z_{k}+\tilde{B} v_{k}, \quad \zeta_{k}=v_{k}, \quad \varepsilon_{k}=z_{k}+\omega_{k}$. With couplings $v_{k}=H \sum_{j \in \mathcal{N}_{k}}\left(\varepsilon_{j}-\varepsilon_{k}\right)$ among these systems, we obtain

$$
\begin{array}{rlrl}
T^{\omega \zeta}: & \dot{z} & =\left(I_{N} \otimes \tilde{A}-L_{\mathcal{G}} \otimes \tilde{B} H\right) z-\left(L_{\mathcal{G}} \otimes \tilde{B} H\right) \omega \\
& \zeta: & \zeta & =-\left(L_{\mathcal{G}} \otimes H\right) z-\left(L_{\mathcal{G}} \otimes H\right) \omega \\
\Delta: & \omega & =\operatorname{diag}\left(\Delta_{k}\right) \zeta .
\end{array}
$$

Since $\mathcal{G}$ is undirected and connected, there exists an orthogonal matrix $U$ such that $U^{\top} L_{\mathcal{G}} U=\Lambda=\operatorname{diag}\left(\lambda_{k}\right)$ and $\lambda_{1}=0$ is the top left diagonal element of $\Lambda$. With the coordinate transformation $\tilde{z}=\left(U^{\top} \otimes I_{N}\right) z, \tilde{\omega}=\left(U^{\top} \otimes I_{N}\right) \omega, \tilde{\zeta}=\left(U^{\top} \otimes I_{N}\right) \zeta$, similarly to [14,29], we obtain the transformed system $T^{\tilde{\omega} \tilde{\zeta}}$ described by $\dot{\tilde{z}}=\left(I_{N} \otimes \tilde{A}-\Lambda \otimes \tilde{B} H\right) \tilde{z}-(\Lambda \otimes \tilde{B} H) \tilde{\omega}, \tilde{\zeta}=$ $-(\Lambda \otimes H) \tilde{z}-(\Lambda \otimes H) \tilde{\omega}$ and $\tilde{\omega}=\left(U^{\top} \otimes I\right) \operatorname{diag}\left(\Delta_{k}\right)(U \otimes I) \tilde{\zeta}$. The system $T^{\tilde{\omega} \tilde{\zeta}}$ consists of the decoupled systems $T_{k}^{\tilde{\omega} \tilde{\zeta}}$ described by $\dot{\tilde{z}}_{k}=\left(\tilde{A}-\lambda_{k} \tilde{B} H\right) \tilde{z}_{k}-\lambda_{k} \tilde{B} H \tilde{\omega}_{k}, \tilde{\zeta}_{k}=-\lambda_{k} H \tilde{z}_{k}-$ $\lambda_{k} H \tilde{\omega}_{k}$. According to the Bounded Real Lemma [36], the system $T_{k}^{\tilde{\omega} \tilde{\zeta}}$ is asymptotically stable and has $\mathcal{H}_{\infty}$ norm less than or equal $\eta>0$, if and only if there exists $X_{k}>0$ such that (40) holds. Furthermore, if $\left\|T_{k}^{\tilde{\omega} \tilde{\zeta}}\right\|_{\infty}<\eta$ for all $k \in \mathcal{N}$, then $\left\|T^{\tilde{\omega} \tilde{\zeta}}\right\|_{\infty}<\eta$ due to the block diagonal structure. Moreover, since $\|U\|_{\infty}=\left\|U^{\top}\right\|_{\infty}=1$ and the maximal singular value of a block diagonal matrix equals the maximum of the maximal singular values of each block, it follows that $\left\|\left(U^{\top} \otimes I\right) \operatorname{diag}\left(\Delta_{k}\right)(U \otimes I)\right\|_{\infty}=\max _{k \in \mathcal{N}}\left\|\Delta_{k}\right\|_{\infty}<1 / \eta$. Asymptotic stability of the perturbed closed loop $T^{\omega \zeta}, \Delta$ is a direct consequence of the Small Gain Theorem [28].

For a given coupling gain $H$ and a nominal system (38), Lemma 2 allows to compute a bound on the $\mathcal{H}_{\infty}$ norm of tolerable uncertainties $\Delta_{k}$. Since (40) is an LMI, the smallest $\eta>0$ can be found via semi-definite programming. Stability of the network is guaranteed for all $\Delta_{k}$ with $\left\|\Delta_{k}\right\|_{\infty}<1 / \eta$. Note that Lemma 2 provides a robust stability result but does not guarantee the desired performance of the heterogeneous network in terms of the exponential decay rate. Nevertheless, the additional coupling term can be expected to improve the convergence speed of the synchronization error significantly, also in groups of non-identical agents.

\section{Conclusion}

The cooperative output regulation problem captures a wide range of practical multi-agent coordination problems. We presented a novel distributed regulator which solves the coordination problem and additionally allows to tune the synchronization error dynamics of the group. A novel coupling term based on the transient state components of each agent allows to impose a desired exponential decay rate on the synchronization error among agents, which leads to a significant improvement of the cooperative behavior of the group in transient phases. Under the novel control law, the group is able to react cooperatively on external disturbances acting 
on individual agents. We presented a vehicle platooning example in order to emphasize the importance of a cooperative reaction on disturbances and illustrate the design procedure and effectiveness of the novel distributed regulator with transient synchronization. Moreover, we outlined a procedure to establish such couplings in groups of non-identical agents with similar dynamics.

\section{Acknowledgements}

We gratefully thank Prof. Jie Chen and Dr. Jie Mei for helpful discussions.

\section{References}

[1] M. Andreasson, D. V. Dimarogonas, H. Sandberg, and K. H. Johansson. Distributed Control of Networked Dynamical Systems: Static Feedback, Integral Action and Consensus. IEEE Trans. Automat. Control, 59(7):1750-1764, 2014.

[2] M. Bartels and H. Werner. Cooperative and Consensus-Based Approaches to Formation Control of Autonomous Vehicles. In Proc. 19h IFAC World Congress, pages 8079-8084, 2014.

[3] D. S. Bernstein. Matrix Mathematics. Princeton Univ. Press, 2009.

[4] M. Chilali and P. Gahinet. $\mathcal{H}_{\infty}$ Design with Pole Place Constraints: An LMI Approach. IEEE Trans. Automat. Control, 41(3):358-367, 1996.

[5] J. A. Fax and R. M. Murray. Information flow and cooperative control of vehicle formations. IEEE Trans. Automat. Control, 49(9):14651476, 2004.

[6] H. F. Grip, T. Yang, A. Saberi, and A. A. Stoorvogel. Output synchronization for heterogeneous networks of non-introspective agents. Automatica, 48(10):2444-2453, 2012.

[7] M. Herceg, M. Kvasnica, C. Jones, and M. Morari. Multi-Parametric Toolbox 3.0. In Proc. European Control Conference, pages 502-510, 2013.

[8] Y. Hong, J. Hu, and L. Gao. Tracking control for multi-agent consensus with an active leader and variable topology. Automatica, 42(7):1177-1182, 2006.

[9] Y. Hong, X. Wang, and Z. Jiang. Distributed output regulation of leader-follower multi-agent systems. Int. J. Robust and Nonlinear Control, pages 48-66, 2013.

[10] J. Huang. Nonlinear Output Regulation: Theory and Application. SIAM, 2004.

[11] J. Huang. Remarks on synchronized output regulation of linear networked systems. IEEE Trans. Automat. Control, 56(3):630-631, 2011.

[12] H. Kim, H. Shim, and J. H. Seo. Output Consensus of Heterogeneous Uncertain Linear Multi-Agent Systems. IEEE Trans. Automat. Control, 56(1):200-206, 2011.

[13] H. W. Knobloch, A. Isidori, and D. Flockerzi. Topics in Control Theory. Birkhäuser, 1993.

[14] Z. Li, Z. Duan, and G. Chen. On $\mathcal{H}_{\infty}$ and $\mathcal{H}_{2}$ performance regions of multi-agent systems. Automatica, 47(4):797-803, 2011.

[15] Z. Li, Z. Duan, G. Chen, and L. Huang. Consensus of Multiagent Systems and Synchronization of Complex Networks: A Unified Viewpoint. IEEE Trans. Circuits and Systems I: Regular Papers, 57(1):213-224, 2010.

[16] J. Löfberg. YALMIP: A Toolbox for Modeling and Optimization in MATLAB. In Proc. CACSD Conference, pages 284-289, 2004.
[17] J. Lunze. Synchronization of Heterogeneous Agents. IEEE Trans. Automat. Control, 57(11):2885-2890, 2012.

[18] W. Ni and D. Cheng. Leader-following consensus of multi-agent systems under fixed and switching topologies. Systems \& Control Letters, 59(3-4):209-217, 2010.

[19] R. Olfati-Saber and R. M. Murray. Consensus problems in networks of agents with switching topology and time-delays. IEEE Trans. Automat. Control, 49(9):1520-1533, 2004.

[20] W. Ren and E. M. Atkins. Distributed multi-vehicle coordinated control via local information exchange. Int. J. Robust and Nonlinear Control, 17(10-11):1002-1033, 2007.

[21] W. Ren and R. W. Beard. Consensus seeking in multiagent systems under dynamically changing interaction topologies. IEEE Trans. Automat. Control, 50(5):655-661, 2005.

[22] L. Scardovi and R. Sepulchre. Synchronization in networks of identical linear systems. IEEE Trans. Automat. Control, 45(11):25572562, 2009.

[23] G. Seyboth and F. Allgöwer. Output synchronization of linear multiagent systems under constant disturbances via distributed integral action. In Proc. American Control Conf. (ACC), pages 62-67, 2015.

[24] G. Seyboth, W. Ren, and F. Allgöwer. Cooperative Control of Linear Multi-Agent Systems via Distributed Output Regulation and Transient Synchronization. arXiv preprint arXiv:1406.0085, 2014.

[25] Y. Su and J. Huang. Cooperative output regulation of linear multiagent systems. IEEE Trans. Automat. Control, 57(4):1062-1066, 2012.

[26] Y. Su and J. Huang. Cooperative output regulation of linear multiagent systems by output feedback. Systems \& Control Letters, 61(12):1248-1253, 2012.

[27] Y. Su and J. Huang. Cooperative output regulation with application to multi-agent consensus under switching network. IEEE Trans. Systems, Man, and Cybernetics - Part B: Cybernetics, 42(3):864875,2012

[28] H. L. Trentelman, A. A. Stoorvogel, and M. Hautus. Control Theory for Linear Systems. Springer Verlag, London, UK, 2001.

[29] H. L. Trentelman, K. Takaba, and N. Monshizadeh. Robust Synchronization of Uncertain Linear Multi-Agent Systems. IEEE Trans. Automat. Control, 58(6):1511-1523, 2013.

[30] S. E. Tuna. LQR-based coupling gain for synchronization of linear systems. arXiv preprint arXiv:0801.3390, 2008.

[31] P. Wieland, J.-S. Kim, and F. Allgöwer. On topology and dynamics of consensus among linear high-order agents. Int. J. Systems Science, 42(10):1831-1842, 2011.

[32] P. Wieland, R. Sepulchre, and F. Allgöwer. An internal model principle is necessary and sufficient for linear output synchronization. Automatica, 47(5):1068-1074, 2011.

[33] J. Xiang, W. Wei, and Y. Li. Synchronized output regulation of linear networked systems. IEEE Trans. Automat. Control, 54(6):1336-1341, 2009.

[34] T. Yucelen and M. Egerstedt. Control of Multiagent Systems under Persistent Disturbances. In Proc. American Control Conference (ACC), pages 5264-5269, 2012.

[35] H. Zhang, F. Lewis, and A. Das. Optimal design for synchronization of cooperative systems: state feedback, observer and output feedback. IEEE Trans. Automat. Control, 56(8):1948-1952, 2011.

[36] K. Zhou and J. C. Doyle. Essentials of Robust Control. Prentice Hall, 1998. 